\title{
Recursos audiovisuales en la docencia a nivel universitaria. El uso del viodeblog como herramienta de comunicación
}

\author{
Eva García MONTERo \\ Universidad Camilo José Cela (España) \\ egarmo@ucjc.edu \\ Laura MELENdo Rodríguez-CARMONA \\ Universidad Camilo José Cela (España) \\ lmelendo@ucjc.edu \\ África Presol Herrero \\ Universidad Camilo José Cela (España) \\ apresol@ucjc.edu
}

\section{Resumen}

En el siguiente texto se relata la aplicación de las nuevas tecnologías en el desarrollo de la actividad docente universitaria. Concretamente en la facultad de comunicación de la UCJC se puso en marcha dos iniciativas en el grado de Publicidad y Relaciona Públicas en las que los alumnos debían emplear el videoblog como herramienta de comunicación.

Palabras clave: Videoblog, comunicación, universidad, publicidad, recurso pedagógico.

\section{Audiovisual resources at university teachers. The use of videoblog as communication tool.}

\begin{abstract}
The following text describes the application of new technologies in the development of university teaching. Specifically in the faculty of communication in UCJC was launched two initiatives in Advertising and Public Relations degree in which students had to use the videoblog as a communication tool.
\end{abstract}

Key words: Videoblog, communication, university, advertising, teaching resource.

\section{Referencia normalizada:}

García Montero, E.; Melendo Rodríguez-Carmona, L. y Presol Herrero, A. (2013) Recursos audiovisuales en la docencia a nivel universitaria. El uso del videoblog como herramienta de comunicación. Historia y Comunicación Social. Vol. 18. Nºspecial Diciembre. Págs. 159-172.

Sumario 1. Introducción 2. Metodologías audiovisuales en el EEES 3. Metodología aplicada: caso práctico 4. Conclusiones 5. Bibliografía 6. Anexo 


\section{Introducción}

La Facultad de Ciencias de la Comunicación de la UCJC, siguiendo el espíritu que trasciende la filosofía enmarcada en el nuevo EEES, y en línea con estos nuevos enfoques de la docencia, está empeñada en buscar nuevos recursos pedagógicos que den mayor protagonismo al alumno en el proceso de aprendizaje. Huyendo de las tradicionales clases magistrales y los trabajos escritos los profesores se han inmerso en las potencialidades que ofrecen las nuevas tecnologías al servicio de la educación.

Este curso 2012-13 dos profesores del título de Publicidad y Relaciones Públicas de la Facultad de Comunicación han implementado el uso del videoblog como práctica de clase en asignaturas, de primer y segundo curso, con contenidos diametralmente opuestos: Psicología de la Comunicación y Documentación.

Los resultados, muy satisfactorios, se describen a continuación:

- Noventa por ciento de entrega de trabajos

- Alta motivación e implicación de los alumnos que reportaron "haberse divertido mucho"

- Asimilación de contenidos y aprovechamiento optimo

- Disminución del plagio al asumir autoría

El objetivo de las asignaturas Psicología de la Comunicación y Documentación era integrar las TIC de una manera eficiente en la labor docente aprovechando todo el potencial que ofrecen para que los alumnos alcancen los objetivos de aprendizaje marcados. A través de una serie de propuestas didácticas los alumnos recurrieron al uso de los blogs, videoblogs y vídeos para asimilar los contenidos propuestos.

En este artículo nos ceñiremos a exponer los resultados alcanzados en el uso del videoblog por parte de los alumnos de ambas asignaturas.

\section{Metodologías audiovisuales en el eees.}

Actualmente conviven diferentes enfoques y formas de entender la enseñanza. En cada uno de ellos el papel de la tecnología es distinto (Fundación Telefónica, 2012). El modelo tradicional está centrado en el profesor, considerándolo una fuente de conocimiento y al alumno un receptor. En este modelo, la tecnología sirve de apoyo a la presentación de los contenidos y a la realización de ejercicios para su aplicación.

El segundo enfoque, centrado en el alumno, implica considerar que se aprende a través de actividades e interrogantes generados desde el alumno y no desde el profesor. En este caso, el profesor es un guía y un facilitador que ha de apoyar al alumno durante su formación. La tecnología es aquí un medio que ayuda a explorar el conocimiento, es una herramienta importante para la búsqueda de información y elaboración de las actividades. 
Por último, el enfoque centrado en la conectividad, se fundamenta en el supuesto de que el aprendizaje no solo tiene una dimensión individual, sino también social. La formación implica aprender en comunidad y ser capaz de contribuir a la construcción del conocimiento. El profesor es un diseñador de espacios de aprendizaje, y la tecnología juega un papel de mediadora en la construcción del conocimiento y la interacción social.

Con el advenimiento de las nuevas tendencias pedagógicas auspiciadas por el Espacio Europeo de Educación Superior (EEES) se han incorporado a la docencia universitaria nuevas plataformas tecnológicas que posibilitan esta interacción del docente con el alumno fuera de la tradicional clase magistral.

Las implicaciones pedagógicas de Internet en la enseñanza y el aprendizaje son muy variadas. Si usamos los recursos de Internet adecuadamente en el aula podemos establecer nuevas vías de comunicación y abrir nuevos canales para encuentros formativos que además aportan una dinámica distinta al día a día de la docencia.

Siguiendo a García Montero et als. (2012), es interesante destacar que Internet como una gran red social permite a los alumnos determinar y controlar su proceso de aprendizaje a la vez que reflexionan sobre el mismo potenciando estrategias metacognitivas que permiten a los alumnos ser mucho más conscientes de los recursos y herramientas que les facilitarán este proceso de adquisición de conocimiento. De esta manera hacen frente a nuevas situaciones de aprendizaje en las que se potencia el trabajo en equipo así como otras estrategias afectivas y sociales que juegan un papel determinante en contextos comunicativos. El trabajo con redes sociales refuerza las relaciones entre los miembros del grupo de clase y se puede aprovechar esta relación de confianza que se establece para proporcionar un entorno libre de estrés. En esta línea, Hossain y Wigand (2004) señalan que las relaciones de confianza que se establecen entre los participantes son de gran importancia en el proceso educativo en tanto que proceso integral.

En términos generales podemos decir que el proceso de enseñar con redes sociales no es una mera transmisión de datos sino que se convierte en un ejercicio colaborativo de compilación, orquestación e integración de información en construcción de conocimiento (Educause, 2009).

Esta orquestación favorece el establecimiento de sinergias para un aprendizaje continuo que se extenderá en el tiempo, es decir que Internet como una gran red favorece y ayuda a configurar el entorno personal de aprendizaje de las personas (Castañeda y Gutiérrez, 2010).

El entorno personal de aprendizaje no está limitado espacialmente ya que Internet permite colaborar con profesores y grupos de alumnos situados en cualquier lugar del mundo que estén interesados en las mismas áreas o proyectos y participar activamente en proyectos conjuntos de creación de conocimiento. Recordando a Lamarck podemos concluir que si la evolución está basada en una interacción constructiva y 
cooperativa de los organismos para adaptarse a los cambios de un entorno dinámico Internet como gran red de redes es un excelente facilitador de esta re-evolución.

Lara (2005) señala cómo frente a otros modelos educativos centrados en la transmisión de contenidos, el constructivismo defiende que el conocimiento es una construcción del ser humano y que se realiza a partir de los esquemas previos que ya posee. Según la pedagogía constructivista, el profesor actúa como mediador, facilitando los instrumentos necesarios para que sea el estudiante quien construya su propio aprendizaje. Cobra, por tanto, especial importancia la capacidad del profesor para diagnosticar los conocimientos previos del alumno y garantizar un clima de confianza y comunicación en el proceso educativo.

Las características propias de los weblogs hacen de esta herramienta un instrumento de gran valor para su uso educativo dentro de un modelo constructivista. Los blogs sirven de apoyo al E-learning, establecen un canal de comunicación informal entre profesor y alumno, promueven la interacción social, dotan al alumno con un medio personal para la experimentación de su propio aprendizaje y, por último, son fáciles de asimilar basándose en algunos conocimientos previos sobre tecnología digital. En este sentido, no hay que olvidar que los alumnos que hoy llegan a la universidad pertenecen a la Generación Red o Net Generation (Oblinger, 2005), que define a los nacidos en la década de 1980 y que han crecido con Internet. Su forma de aprender tiene que ver con esta naturaleza generacional y requiere de nuevos enfoques educativos.

Está constatado que en estos momentos las redes sociales se han convertido en un medio ineludible para los comunicadores. Bien hablemos de publicidad, periodismo o comunicación audiovisual los profesionales de la comunicación recurren a Internet para difundir sus mensajes, los cuáles, a su vez, deben adaptarse a esta realidad tanto en producción como en tecnología y modelos de difusión.

Para los profesionales de la comunicación Internet no es sólo un medio, sino también un canal capaz de emular las funciones de todos los otros medios. Si analizamos las cifras de consumo de vídeo en Internet nos damos cuenta que la televisión, si la asimilamos a los contenidos audiovisuales, no solo no ha muerto, sino que podemos decir que vive su mejor momento. El consumo de vídeo a través de Internet se ha vuelto imparable, sobre todo por el impulso de los smartphone y las tablets.

El informe de IAB sobre video marketing en 2011, (gráficos 1 y 2) señala que la mitad de los usuarios habituales de Internet (53\%) declara ver vídeos a través de este canal diariamente. Cuando el rango temporal se extiende a la semana, la proporción se eleva hasta el $78 \%$. Pero sobre todo es significativo el impacto sobre el tiempo dedicado a Internet. En promedio, los encuestados estiman que una de cuatro horas de navegación se destina a ver vídeo, siendo los internautas más jóvenes (18-30 años) quienes lideran el consumo con el $35 \%$ de su tiempo dedicado a esta actividad; doblando al de los usuarios de entre 41 y 55 años (19\%). 
Gráfico 1: ¿Con qué frecuencia ves vídeos (películas, noticias, etc) en Internet?

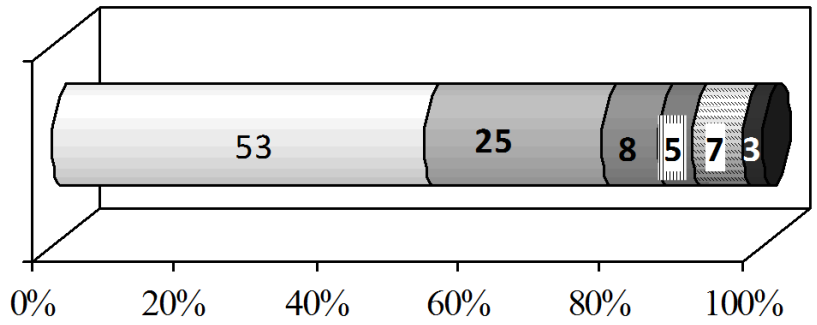

$\begin{array}{ll}\square \text { Diariamente } & \square \text { Al menos una vez por semana } \\ \square \text { Al menos una vez cada } 15 \text { días } & \square \text { Al menos una vez al mes } \\ \text { Con menor frecuencia } & \square \text { Nunca }\end{array}$

Fuente: elaboración propia con datos de IAB (2011)

Gráfico 2: Pensando en el uso de Internet que realizas normalmente, aproximadamente, ¿qué porcentaje de tiempo dirías que dedicas semanalmente a ver vídeos online, ya sean películas, series, videos de Youtube...)?

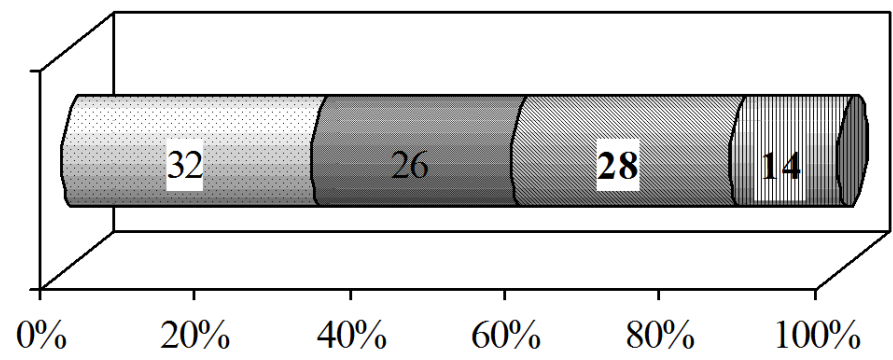

$\square$ Hasta $10 \% \quad \square 11-25 \% \quad \square 26-50 \% \quad \square$ Más de $50 \%$

Fuente: elaboración propia con datos de IAB (2011)

De manera que podemos constatar que no sólo se ha producido una oferta de vídeos, la utilización del vídeo como formato de comunicación y como herramienta clave en la estrategia de marketing de contenidos continua en ascenso, sino que también ha aumentado la demanda, nuestro consumo se sitúa entre los más altos de Europa ${ }^{1}$.

Uno de los motores de crecimiento del consumo de vídeo online es, sin lugar a dudas, la universalización del uso de las redes sociales, que favorece la difusión de

1 http://www.comscore.com/Press_Events/Press_Releases/2011/6/Germany_Leads_Europe_in_ Online_Vídeo_Viewing 
este tipo de contenido. El mismo informe IAB señala que, por muy poca diferencia, el correo electrónico continúa siendo el medio más utilizado a nivel doméstico para distribuir vídeo. Seis de cada diez encuestados dice compartir vídeo a través de este medio todos o casi todos los días, frente al $51 \%$ que hace lo propio mediante las redes sociales (gráfico 3). Aunque a distancia, otros medios sociales relevantes, como los blogs o los foros, también están siendo utilizados con profusión para compartir vídeo: el $28 \%$ y $42 \%$ utiliza los blogs y foros, respectivamente, con esta finalidad al menos una vez cada 15 días. Los encuestados estiman que, del total del tiempo invertido en el consumo de vídeo, casi un cuarto (22\%) corresponde al que comparten sus contactos en redes sociales como Facebook o Twitter. Asimismo, los datos confirman que desplegar una buena estrategia de vídeo marketing en redes sociales resulta eficaz para llegar al consumidor: casi dos de cada diez minutos (17\%) del vídeo consumido, corresponden a lo que comparten empresas, páginas de fans y grupos que se siguen en estas plataformas. Un 28\% comparte vídeos online de spots de televisión al menos semanalmente (gráfico 4). Un porcentaje similar (25\%) también comparte otros tipos de vídeos de empresas y marcas.

Gráfico 3: ¿Con qué frecuencia utilizas los siguientes medios para compartir con tus contactos (familia, amigos, compañeros...) videos que te gustan? \% Diario/casi diario. Respuesta múltiple posible.

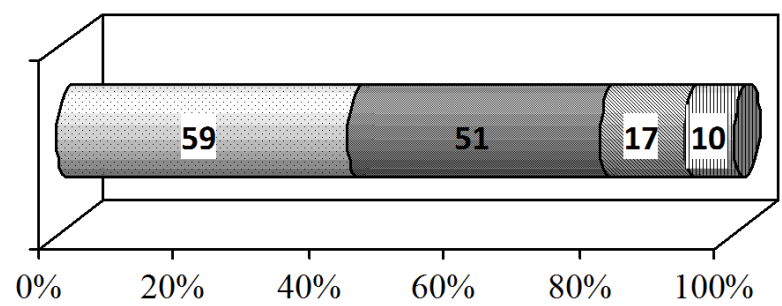

$\square$ Correo $\square$ Redes Sociales (Facebook, T uenti, Twitter...) $\square$ Foros $\square$ Blogs

Fuente: elaboración propia con datos de IAB (2011).

Gráfico 4: ¿Con qué frecuencia sueles compartir los siguientes videos online? \% semanalmente. Respuesta múltiple posible. 


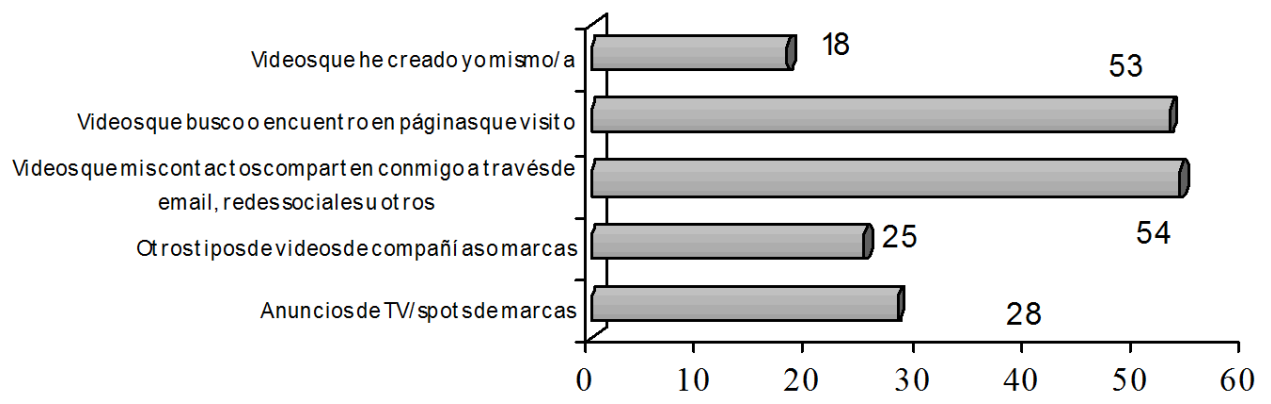

Fuente: elaboración propia con datos de IAB (2011)

Gráfico 5: Pensando en tus hábitos de uso de Internet y en el tiempo total que inviertes viendo vídeos en Internet, ¿qué porcentaje aproximado de ese tiempo total lo dedicas a vídeos a los que has accedido de las siguientes formas?

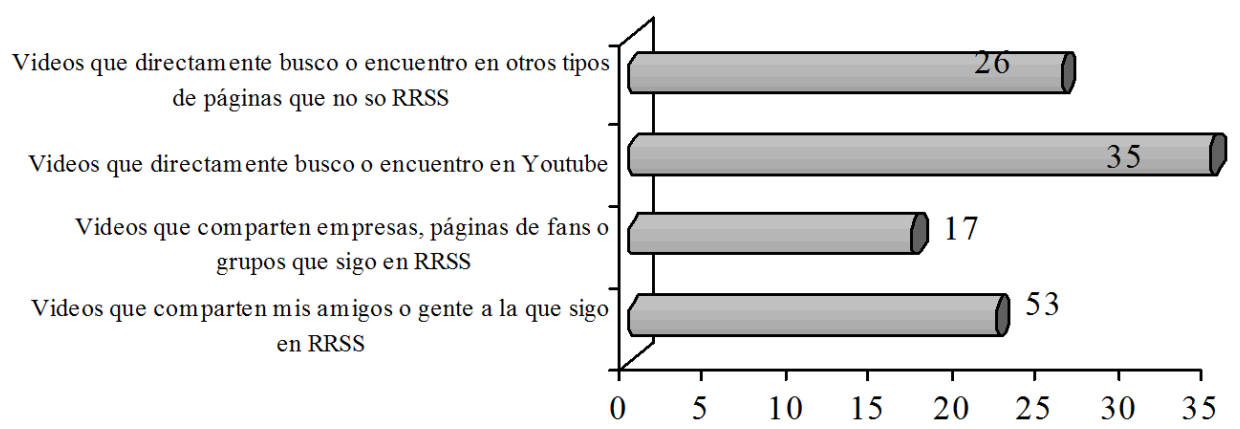

Fuente: elaboración propia con datos de IAB (2011)

Podemos considerar entonces que la audiovisualización de la web es un hecho. Se trata de un fenómeno con una alta penetración entre todos los targets, que representa una de las actividades a la que mayor tiempo se dedica en Internet. Además, buena parte de este consumo es social; es decir, gran parte del tiempo que se dedica al visionado de vídeos corresponde a contenidos que han sido compartidos o recomendados por terceros, principalmente, a través del correo electrónico y de los medios sociales.

Es así, que los alumnos de comunicación no pueden ser ajenos a esta realidad. Deben manejar los recursos audiovisuales como manejaban antaño los recursos orales y escritos pues formarán parte del día a día de su profesión. 


\section{Metodología aplicada: caso práctico.}

Un videoblog o Vlog es una bitácora o blog que se constituye sobre la base de clips de vídeo ordenada cronológicamente y actualizados periódicamente. Puede ser individual o grupal. Los clips de vídeo pueden ser registros propios o de otros usuarios, incluidos mediante un vínculo. Dependiendo de lo que se quiera comunicar, el vídeo puede ir solo o acompañado de un comentario textual. También admite que otros usuarios comenten los contenidos expuestos.

Los comentarios son una parte fundamental en la estructura de un blog o videoblog ya que se trata de una herramienta que promueve el diálogo.

El término bitácora hace referencia a notas personales, semejantes a un diario de vida, aunque no es restrictivo a este tipo de material y se pueden encontrar videoblogs de los más diversos contenidos.

El uso de un videoblog en el proceso educativo aporta una serie de ventajas:

- Ofrece mayor dinamismo y motivación en el trabajo con los alumnos.

- Promueve el protagonismo del alumno.

- Mejora la presentación y la comprensión de cierto tipo de información. Amplía los lenguajes a los que tiene acceso y maneja el alumno.

- Junto con la pizarra digital es un recurso positivo para currículos de enseñanza superior.

- Presenta nuevas formas de afrontar el trabajo grupal mediante un videoblog multiusuario con entradas independientes.

- Ofrece una mejora continua en la evaluación permanente del proceso de aprendizaje.

- Permite aprovechar los múltiples materiales didácticos en formato vídeo que se pueden encontrar en Internet y asociar al videoblog.

- Presenta todas las ventajas que conlleva el uso didáctico de Internet. Espacio social rico en fuentes de información orientado al trabajo autónomo y colaborativo, crítico y creativo, la expresión personal, investigar y compartir recursos, crear conocimiento y aprender.

- Facilita un aprendizaje más autónomo, mayor participación en las actividades grupales, hay más interés y motivación.

- Permite elaborar materiales, compartirlos y someterlos a comentarios de los lectores.

- Libera al docente de ser la fuente principal de información para dedicarse a dirigir el trabajo de los alumnos.

- Mejora competencias digitales como buscar, seleccionar, procesar y comunicar la información.

- Estilo informal y espontáneo pero respetuoso con las normas ortográficas, gramaticales y de expresión oral.

- Permite acceder a nuevos entornos y situaciones de aprendizaje.

- Promueve la autoevaluación de los alumnos. 
Existe una gran cantidad de material audiovisual consolidada en la web que nos permite realizar interesantes búsquedas. El constante avance tecnológico promete vídeos cada día de mejor calidad y mayor duración, descargados prácticamente sin demora alguna. Por otra parte, como medio de comunicación audiovisual, el videoblog es el desarrollo lógico del blog, en el cual el texto viene a ser un apoyo o complemento de unos contenidos registrados con una cámara de vídeo o en muchos casos con una Webcam.

Las prácticas que se pidieron a los alumnos consistían en crear y administrar contenidos en un videoblog. En concreto el tema que se manejaba era distinto para cada una de las asignaturas.

Las pautas que se dieron a los alumnos en la asignatura de Psicología de la Comunicación fueron las siguientes:

Para el tema 2 (Actitudes) los alumnos harán un videoblog. El objetivo o contenido del mismo será plantear la diferencia entre estereotipos, prejuicios y conductas discriminatorias. Debe establecerse la relación con los tres componentes de las actitudes (cognitivo, evaluativo, conativo).

Se puede ampliar información acerca de prejuicios y estereotipos en el apartado de percepción social del tema 4 de esta misma asignatura.

Observaciones:

El videoblog:

- Se puede hacer en grupo, como máximo puede haber tres componentes.

- No debe durar más de cinco minutos

- Se subirá a youtube y se moderarán los comentarios

- Para optar a evaluación uno de los miembros del grupo lo subirá a la Blackboard en su nombre y hará constar el nombre del resto de los componentes

- La evaluación de esta práctica tendrá tres partes: la evaluación del resto de compañeros $(20 \%)$, número de visitas en youtube y comentarios $(20 \%)$ y evaluación del profesor $(60 \%)$

Mientras que las pautas que se dieron a los alumnos en la asignatura de Documentación fueron estas otras:

Para el tema 1 (Documentación) los alumnos realizarán en grupo un videoblog.

El objetivo será mostrar al mundo su visión de lo que entienden por Documentación, respondiendo a qué es Documentación entendida como ciencia, por qué es importante y para qué sirve.

Observaciones:

El videoblog:

- Se puede hacer en grupo, como máximo puede haber cinco componentes. 
- No debe durar más de un minuto

- Se subirá a youtube El video más visitado obtendrá un punto directamente aplicable sobre la calificación final de la práctica

- Para optar a evaluación uno de los miembros del grupo subirá una captura de pantalla a la Blackboard con su nombre y hará constar el nombre del resto de los componentes

- La evaluación de esta práctica tendrá tres partes: la evaluación del resto de compañeros $(20 \%)$ y la evaluación del profesor $(80 \%)$

Para el tema 5 (Fuentes de información. Fiabilidad) los alumnos realizarán en grupo un videoblog.

El objetivo se basará en contar una historia sobre el cambio climático en 30 segundos a través de un video de gran alcance en el que hay que crear conciencia, estimular la acción para un cambio y/u ofrecer nuevas soluciones para este problema mundial. Connect4Climate (C4C), una iniciativa de cambio climático en marcha desde el 2011 por el Banco Mundial, el Fondo para el Medio Ambiente Mundial y el Ministerio italiano de Medio Ambiente.

Observaciones:

El videoblog:

- $\quad$ Se puede hacer en grupo, de hasta 5 personas, edades 18-35.

- No debe durar más de treinta segundos

- Se subirá a la página oficial de Connect4Climate (C4C)

- Para optar a evaluación uno de los miembros del grupo subirá una captura de pantalla a la Blackboard con su nombre y hará constar el nombre del resto de los componentes

- La evaluación de esta práctica dependerá de la evaluación del profesor $(100 \%)$

- Se presentará en clase el día 24 de mayo

En los siguientes enlaces se pueden consultar diversos ejemplos de los trabajos que realizaron los alumnos en cada una de las asignaturas:

- Vídeo 1: ejemplo de la asignatura de Psicología de la comunicación. Disponible en http://www.youtube.com/watch?v=ELW9_g7XpLU

- Vídeo 2: ejemplo de la asignatura de Psicología de la comunicación. Disponible en http://www.youtube.com/watch? $\mathrm{v}=$ cceKqqOox9Y

- Vídeo 3: ejemplo de la asignatura de Documentación. Disponible en http:// www.youtube.com/watch? $\mathrm{v}=\mathrm{xPHeq} 9 \mathrm{~V} 1 \mathrm{ZUc}$

- Vídeo 4: ejemplo de la asignatura de Documentación. Disponible en https:// www.youtube.com/watch?v=8d-3EsPQoMw

La experiencia tuvo un gran éxito cuantitativo y cualitativo así como de consecución de objetivos de aprendizaje: 
- Más de 90\% de entrega de trabajos en ambas asignaturas.

- Incremento de la motivación e implicación de los alumnos que reportaron "haberse divertido mucho". Permite un estilo informal y espontáneo aunque respetuoso con la ortografía, la gramática y la expresión oral.

- Asimilación de contenidos y aprovechamiento óptimo que se comprobó con preguntas aleatorias días posteriores en clase.

- Disminución del plagio, uno de los problemas encontrados en los trabajos de investigación del curso anterior, al asumir autoría.

- Aumento del manejo de los lenguajes a los que tiene acceso el alumno: "oral" al ser protagonistas de los vídeos y narrarlos en primera persona, "escrito" en la moderación de comentarios y "audiovisual" en la realización técnica.

- Entrenamiento de las habilidades grupales y el trabajo colaborativo. Inexcusable la organización entre los miembros, no pueden hacer cada uno su parte, todos deben estar en todo.

- Mejora de competencias digitales como buscar, seleccionar, procesar y comunicar la información.

\section{Conclusiones.}

El uso del videoblog no es sólo interesante para nuestros alumnos de la Facultad de Comunicación como herramienta efectiva desde un punto de vista pedagógico o educativo. No debemos olvidar el desarrollo de Internet como canal de comunicación en si mismo. Internet no sólo no sustituye a otros medios si no que, en algunos casos, los sustenta (véase el nuevo renacer de la televisión) y en concreto la audiovisualización de la Red de redes hacen especialmente necesario que los alumnos de comunicación desarrollen competencias relacionadas con la producción y difusión de contenidos audiovisuales.

Para la formación efectiva de un alumno en la Sociedad de la Información y del Conocimiento (SIC), la educación debe asumir el reto de la alfabetización digital de manera que puedan hacer un uso libre y responsable de los recursos en Internet. Según Trejo Delarbre (2005), en este nuevo entorno el ciudadano requiere de destrezas específicas para su supervivencia: «La capacitación, no sólo para encontrar información y saber discriminar entre ella, sino también para colocar contenidos en las redes informáticas, se ha convertido en requisito indispensable en la formación cultural, la competitividad laboral y las opciones sociales de las personas».

La red debe ser utilizada como medio y objeto de conocimiento, de tal manera que el docente debe desarrollar didácticas adaptadas a la demanda de formación continua y a distancia en un nuevo modelo de aprendizaje centrado en el alumno. La enseñanza con blogs responde a esta nueva realidad social, puesto que puede contribuir a gestionar la sobreabundancia de información y a extraer sentido de la misma al tiempo que el alumno se convierte en el protagonista de su aprendizaje. 
Los blogs y videoblogs, como la educación, son por su propia naturaleza procesos de comunicación, de socialización y de construcción de conocimiento. Una de sus características más importantes es la capacidad de interactividad, que permite que el webblog pase de ser un monólogo a un diálogo en una invitación constante a la conversación. De esta forma, el alumno puede recibir el feedback de otros participantes en el debate y tomar mayor conciencia de su propio aprendizaje (Ferdig y Trammell, 2004). Además de observar las conversaciones que tienen lugar en su propio blog a través de los comentarios que recibe, o ver las referencias a su blog con los trackbacks, el alumno puede también seguir la evolución del debate de los blogs donde haya dejado sus comentarios, gracias a los servicios de agregación de contenidos. Además, la creación de videoblogs colectivos permite desarrollar capacidades de trabajo colaborativo a través de la distribución de funciones en el grupo y del establecimiento de un modelo de tutoría mutua entre sus integrantes.

Otra cuestión a considerar es la determinación de autoría que elimina el plagio tan frecuente en los trabajos universitarios. El hecho de que el Weblog sea público genera un sentido de responsabilidad en los alumnos, quienes ponen en juego su propia reputación en la Red y valoran su trabajo por encima de lo que podría ser un requisito académico dirigido exclusivamente al profesor (Lankshear y Knobel, 2003). El profesor deja de ser el único destinatario de la producción del alumno y pasa a ser uno más entre todos los potenciales lectores de la Red. Este carácter público e interactivo es fundamental para entender el potencial socializador y motivador de los blogs y videoblogs.

\section{Referencias bibliográficas.}

Capítulos o artículos en libros o revistas en papel

CASTAÑEDA, L y GUTIERREZ, I. (2010): Redes sociales y otros tejidos online para conectar personas, en CASTAÑEDA, L et al. Aprendizaje con redes sociales. Eduforma. Sevilla.

GARCÍA MONTERO Eva, DE LA MORENA TABOADA, Marián y MELENDO RODRÍGUEZ-CARMONA, Laura. (2012): Twitter a examen. Las redes sociales como punto de encuentro entre docentes y alumnos. Revista Estudios sobre el Mensaje Periodístico

HOSSAIN, L. y WIGAND, R. (2004). Ict enabled virtual collaboration through trust. Journal of Computer-mediated Communication 10, no 1.

LANKSHEAR, C. y KNOBEL, M. (2003) Do-It-Youself Broadcasting: Writing Weblogs in a Knowledge Society, Annual Meeting of the American Educational Research Association. Chicago: AERA.

TREJO DELABRE, R. (2005) La persona en la Sociedad de la Información, citado en La Sociedad de la Información en el siglo XXI: un requisito para el desarrollo 
(Vol.2). Reflexiones y conocimiento compartido. Secretaría de Estado de Telecomunicaciones y para la Sociedad de la Información.

Artículos en publicaciones web

EDUCASE (2009) 7 Things you should know about Personal Learning Environments en Educase. 12 de mayo de 2009. Disponible en http://net.educause.edu/ir/library/ pdf/ELI7049.pdf. Consultado el 20 de julio de 2012.

FERDIG, R. E., y TRAMMELL, K. D. (2004) Content Delivery in the 'Blogosphere en The Journal On line. Disponible en http://www.thejournal.com/magazine/ vault/A4677.cfm. Consultado el 20 de julio de 2012.

FUNDACIÓN TELEFÓNICA (2011) Universidad 2020. Papel de las TIC en el nuevo entorno socioeconómico. Madrid. Disponible en http://www.fundacion.telefonica.com/es/que hacemos/conocimiento/publicaciones/detalle/153. Consultado el 6 de junio de 2013 .

IAB. (2001) Vídeo marketing y publicidad en vídeo online: aproximación desde la perspectiva del usuario. en Interactive Advertising Bureau. Disponible en www. iabspain.net Consultado el 6 de diciembre de 2011

LARA, T (2005) Blogs para educar. Usos de los blogs en una pedagogía constructivista en Telos, cuadernos de comunicación e innovación. Disponible en http:// sociedadinformacion.fundacion.telefonica.com/telos/articulocuaderno.asp@idarticulo $=2 \&$ rev $=65 . \mathrm{htm}$. Consultado el 7 de junio de 2013 .

OBLINGER, D. G. y OBLINGER, J. L. (2005) Educating the Net Generation en Educause. Libro electrónico. http://www.educause.edu/books/educatingthenetgen/5989. Consultado el 7 de junio de 2013.

\section{Las autoras}

Eva García Montero es doctora en Ciencias Sociales por la Universidad Camilo José Cela, licenciada en Psicología Industrial por la UNED y licenciada en Publicidad y Relaciones Públicas por la Universidad Complutense. También cuenta con un título de Especialista en Gestión Publicitaria por esta última Universidad. Ha desarrollado su carrera profesional en agencias de publicidad (McCann-Erickson y J. Walter Thompson) y departamentos de marketing de empresas, lo que compagina con su labor como docente en la Universidad Camilo José Cela de Madrid donde imparte asignaturas de Relaciones Públicas y Psicología de la Comunicación. Es profesora invitada en Plantjin I Hogeschool Amberes (Bélgica), Artevelde Hogeschool Gante (Bélgica) y Erasmus Hogeschool Bélgica. Sus líneas de investigación y conferencias están vinculadas con la inteligencia emocional, las técnicas de comunicación oral y la aplicación de las TIC a la docencia.

Laura Melendo Rodríguez-Carmona es Doctora en Ciencias de la Información y colabora como docente en la Universidad Camilo José Cela en la Facultad de Ciencias de la Comunicación. En EAE Business School, es líder del Módulo de Planificación de Medios en el Máster de Comunicación Corporativa. Imparte la asignatura 
de Comunicación y Moda en el grado de Diseño de Moda de ESNE. Tiene experiencia en agencias de publicidad y agencias de medios: Mindshare, Carat, The Media Partnership, Amiriatis Puris Lintas y Orange Media. Sus líneas de investigación giran en torno a los medios publicitarios y las nuevas tecnologías aplicadas a la docencia universitaria.

África Presol Herrero es doctora en Publicidad y Relaciones Públicas por la Universidad Camilo José Cela. Licenciada en Ciencias de la Información en la rama de Publicidad y Relaciones Públicas por la Universidad Complutense de Madrid (UCM). CAP por el Instituto de Ciencias de la Educación en la UCM. Especialista en Diseño Gráfico actualmente es docente en la Facultad de Comunicación de la Universidad Camilo José Cela donde imparte las asignaturas de Creatividad, Documentación, Comunicación e imagen corporativas, Teoría y práctica de la Publicidad y Diseño Gráfico Publicitario. En su bagaje profesional cuenta con haber trabajado para cuentas como Buena Vista International-Disney, Publirama, Talante de Comunicación y Oracle. Sus líneas de investigación giran en torno al análisis del mensaje publicitario, al desarrollo de técnicas de estimulación de la creatividad y a la publicidad On line en concreto a las nuevas formas de comunicación en los medios. 